\title{
CPEB3 is associated with human episodic memory
}

\section{Christian Vogler 1,2, Klara Spalek ${ }^{1,2}$,Amanda Aerni ${ }^{3}$, Philippe Demougin ${ }^{1,2}$, Ariane Müller ${ }^{1,2}$, Kim-Dung Huynh ${ }^{1,2}$, Andreas Papassotiropoulos ${ }^{1,2}$ and Dominique J.-F. de Quervain ${ }^{3,4 *}$}

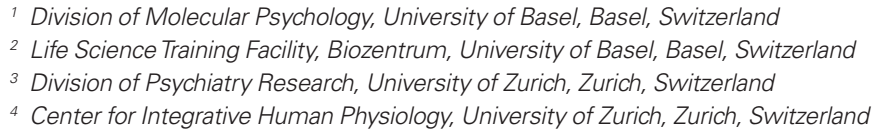

\section{Edited by:}

Isabelle Mansuy, University of Zurich,

Switzerland

\section{Reviewed by:}

Andre Fischer, European Neuroscience Institute, Germany

Irini Skaliora, Biomedical Research

Foundation of the Academy of Athens,

Greece

Satoshi Kida, Tokyo University of

Agriculture and Technology, Japan

*Correspondence:

Dominique J.-F. de Quervain,

Division of Psychiatry Research,

University of Zurich, Lenggstr. 31,

CH 8032 Zurich, Switzerland.

e-mail:quervain@bli.uzh.ch
Cytoplasmic polyadenylation element-binding (CPEB) proteins are crucial for synaptic plasticity and memory in model organisms. A highly conserved, mammalian-specific short intronic sequence within CPEB3 has been identified as a ribozyme with self-cleavage properties. In humans, the ribozyme sequence is polymorphic and harbors a single nucleotide polymorphism that influences cleavage activity of the ribozyme. Here we show that this variation is related to performance in an episodic memory task and that the effect of the variation depends on the emotional valence of the presented material. Our data suggest a role for human CPEB3 in human episodic memory.

Keywords: CPEB3, emotional, episodic, memory, polymorphism

\section{INTRODUCTION}

Learning and memory requires biochemical and structural changes at specific neuronal synapses and RNA-binding molecules are thought to play an important role in inducing and maintaining such synaptic changes (Klann and Dever, 2004). The cytoplasmic polyadenylation element (CPE) promotes cytoplasmic polyadenylation of dormant mRNAs that have small poly(A) tails. In response to synaptic stimulation the poly $(\mathrm{A})$ tails are elongated and translation can ensue (Huang et al., 2003; Mendez et al., 2000). This mechanism allows for protein dependent cellular and synaptic transformations. Cytoplasmic polyadenylation element-binding (CPEB) proteins constitute a family of regulators of mRNA translation and have been related to synaptic plasticity and memory formation in model organisms (Richter, 2007). In Aplysia, a neuron-specific CPEB isoform is upregulated locally at activated synapses and is required for the maintenance of long-term facilitation, possibly through a prion-like mechanism (Si et al., 2003a,b). The Drosophila CPEB2 protein Orb2 is required for long-term courtship memory (Keleman et al., 2007). CPEB1 knockout mice do not show detectable defects in longterm memory formation, however, extinction of hippocampaldependent memories is reduced (Berger-Sweeney et al., 2006). CPEB3, another member of the CPEB family, which has been also related to synaptic plasticity, is present in the human hippocampus and is co-localized with the synaptic marker synaptophysin (Huang et al., 2006).

Recently, a genome-wide search for ribozymes (i.e., catalytic RNAs) in the human genome identified a highly conserved, mammalian-specific short intronic sequence within $C P E B 3$ as a ribozyme with self-cleavage properties possibly regulating $C P E B 3$ translation (Salehi-Ashtiani et al., 2006). A 72-nt sequence upstream of the third CPEB3 exon is sufficient to carry out self-cleavage, forms the core of the ribozyme, and harbors a single nucleotide polymorphism (SNP rs11186856 according to dbSNP; $U$ to $C$ substitution at position 36 of the core sequence; Figure 1). Compared to the ancestral sequence, the rare $C$ containing sequence exhibits more than twofold rate of self-cleavage. A higher rate of selfcleavage can result in an increased number of truncated CPEB3 pre-mRNAs and ultimately in decreased CPEB3 protein expression (Salehi-Ashtiani et al., 2006).

Given the presumed role of CPEB proteins in synaptic plasticity and memory in model organisms and the recent evidence suggesting the existence of a mammalian conserved, functional $C P E B 3$ ribozyme, we aimed at investigating the role of $C P E B 3$ in human memory. The fact that the human $C P E B 3$ ribozyme sequence harbors a SNP affecting the ribozyme functioning enabled us to adopt a behavioral genetics approach by correlating inter-individual variability in memory performance with individual patterns of genetic variability within $C P E B 3$. We have focused on episodic memory as assessed by verbal memory recall tests $5 \mathrm{~min}$ and $24 \mathrm{~h}$ after learning (de Quervain et al., 2003).

\section{MATERIALS AND METHODS STUDY SUBJECTS AND MEMORY TESTING}

Memory testing and genotyping were performed in 333 healthy young Swiss subjects (232 females, 101 males; median age: 22 years, range: $18-45$ years). After complete description of the study to the subjects, written informed consent was obtained. The ethics committee of the Canton of Zurich, Switzerland approved the study protocol. Subjects viewed six series of five semantically unrelated German nouns presented at a rate of one word per second with the instruction to learn the words for immediate free 


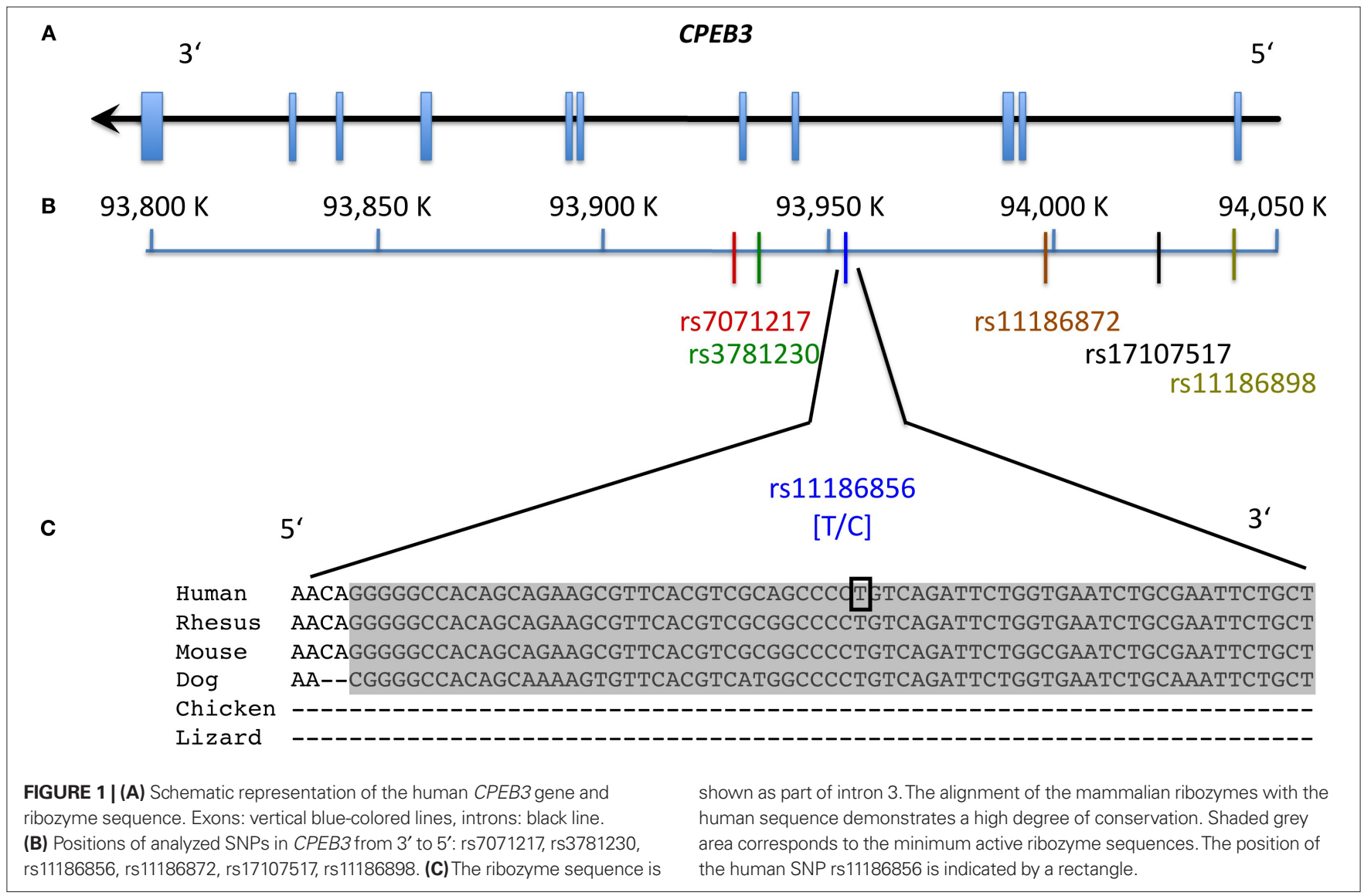

recall after each series. The words were taken from the collections of Hager and Hasselhorn (1994) and consisted of 10 neutral words such as "angle" [valence: $0.2 \pm 0.3$ (mean \pm SEM), rating on a scale from -10 (most negative valence) to +10 (most positive valence) ], 10 positive words such as “joy" (valence: $7.9 \pm 0.4$ ) and 10 negative words such as "poverty" (valence: $-8.5 \pm 0.3$ ). Words were recalled immediately after every series of five words. Five minutes and $24 \mathrm{~h}$ after presentation of these words, subjects underwent unexpected delayed free-recall tests for the same words. The design of an unexpected free-recall test was chosen to prevent confounding factors such as unbalanced reiteration of the learned words.

\section{GENOTYPING AND STATISTICS}

We collected 18 -ml blood $\left(2 \times 9\right.$ Monovette ${ }^{\circledast}$ EDTA tubes; Sarstedt, Germany) from each donor by venous puncture. Genomic DNA was extracted using the QIAamp DNA blood maxi kit (Qiagen, Hilden, Germany). Information on polymorphic sites was derived from the database of single nucleotide polymorphisms (dbSNP) established by the National Center for Biotechnology Information ${ }^{1}$. Genotyping was done with Pyrosequencing on a PyroMark ${ }^{\mathrm{TM}}$ ID System (Biotage, Uppsala, Sweden). SNPs selected for genotyping were: rs7071217, rs3781230, rs11186856, rs11186872, rs17107517, rs11186898. Primers for rs7071217

${ }^{1}$ http://www.ncbi.nlm.nih.gov/SNP/index.html were: 5'-CTT GCA GAA GAG GAT TCA CAT CA-3' (forward, 5'-biotinylated), 5'-GTG TGT CTG CCT GAA GGA ATG-3' (reverse), 5'-GTG TAT CTC AGA AAA GAC CT-3' (sequencing). Primers for rs3781230 were: $5^{\prime}$-TTT TAG AAA AGA AAC CTT TAC ACA-3' (forward, 5' -biotinylated), 5'-ATG CAA AAA AAT GGA GAA AAC AA-3' (reverse), 5' -TTA ACA TTT TGA TTT ACA GT-3' (sequencing). Primers for rs 11186856 were: $5^{\prime}$ CAG AAT TCG CAG ATT CAC CA-3' (forward, 5'-biotinylated), 5' TGC ATA AAA GCT GCT GCT TAG T-3' (reverse), 5'-TTC ACG TCG CAG CCC-3' (sequencing). Primers for rs11186872 were: 5'-CCG CCC TTC CAC AGA AAT T-3' (forward), 5'-TGC AAG GTC AAG CAA GAT AGA CTG-3' (reverse, 5' -biotinylated), 5'-CCT GAG CTA AGT CCA ATG-3' (sequencing). Primers for rs17107517 were: $5^{\prime}$-CCA GAT GTC ATA AAA TGG AGG TAA-3' (forward, 5'-biotinylated), 5'-GGC ATG ATA GCT CCA TTT AAG AGA-3' (reverse), 5'-CAC CCT CTG CTG GGA-3' (sequencing). Primers for rs 11186898 were: $5^{\prime}$-AAA CTA CCA GCG TCC TAC CAA TCA-3' (forward, 5' -biotinylated), 5'-GGA AAG AGC TGT CGG CTC AAA-3' (reverse), 5'-CGA ATG CGT TTT TTG-3' (sequencing). Genotype distributions were in Hardy-Weinberg equilibrium. Comparison of mean memory performances between genotype groups was done with ANCOVA controlling for gender and education (SPSS 11.0.4) ${ }^{2}$. Statistical significance was assumed when $P \leq 0.05$.

${ }^{2}$ http://www.spss.com 


\section{RESULTS}

In a population of 333 healthy young individuals who underwent a verbal episodic memory task, 49 homozygous carriers of the rare $C$ allele-containing ribozyme sequence showed significantly poorer delayed memory recall performance ( 5 min after presentation of 30 semantically unrelated words, see Materials and Methods) than $T$ allele carriers of SNP rs11186856 (7.5 \pm 0.4 recalled words 5 min after learning (mean \pm SEM) vs. 9.0 \pm 0.2 , respectively; $F=9.2 ; \mathrm{df}=1 ; P=0.003$; Figure 2 ). Nearby SNPs in the same haplotype block were also significantly associated with episodic memory performance (Figure 2). In accordance with the haplotype structure of this genomic region, significances dropped to chance levels at chromosomal positions 93822581 (rs1935864) and 94052611 (rs11186914). We did not observe an allele-dose effect, i.e., the reduction of episodic memory performance was restricted to homozygous $C$ allele carriers of SNP rs11186856 (CC genotype group, $n=49: 7.5 \pm 0.4$ words; $C T$ genotype group, $n=140: 9.0 \pm 0.2$; TT genotype group, $n=144$ : $9.0 \pm 0.2 ; F=4.6 ; \mathrm{df}=2 ; P=0.011)$. Furthermore, there was no genotype-dependent difference in immediate recall performance $(P=0.9)$.

Homozygous carriers of the rare $C$ allele of SNP rs11186856 also showed significantly poorer delayed memory recall performance $24 \mathrm{~h}$ after word presentation than $T$ allele carriers $(6.7 \pm 0.5$ recalled words (mean \pm SEM) vs. $7.8 \pm 0.2$, respectively; $F=4.0$; $\mathrm{df}=1 ; P=0.045)$.

The 30 words presented during the episodic memory task differed in their emotional valence (10 words with positive valence, 10 words with negative valence, 10 words with neutral valence; see Materials and Methods). We therefore ran the genetic association analysis of CPEB3 SNPs in each of the three groups separately. Homozygous carriers of the rare $C$ allele-containing

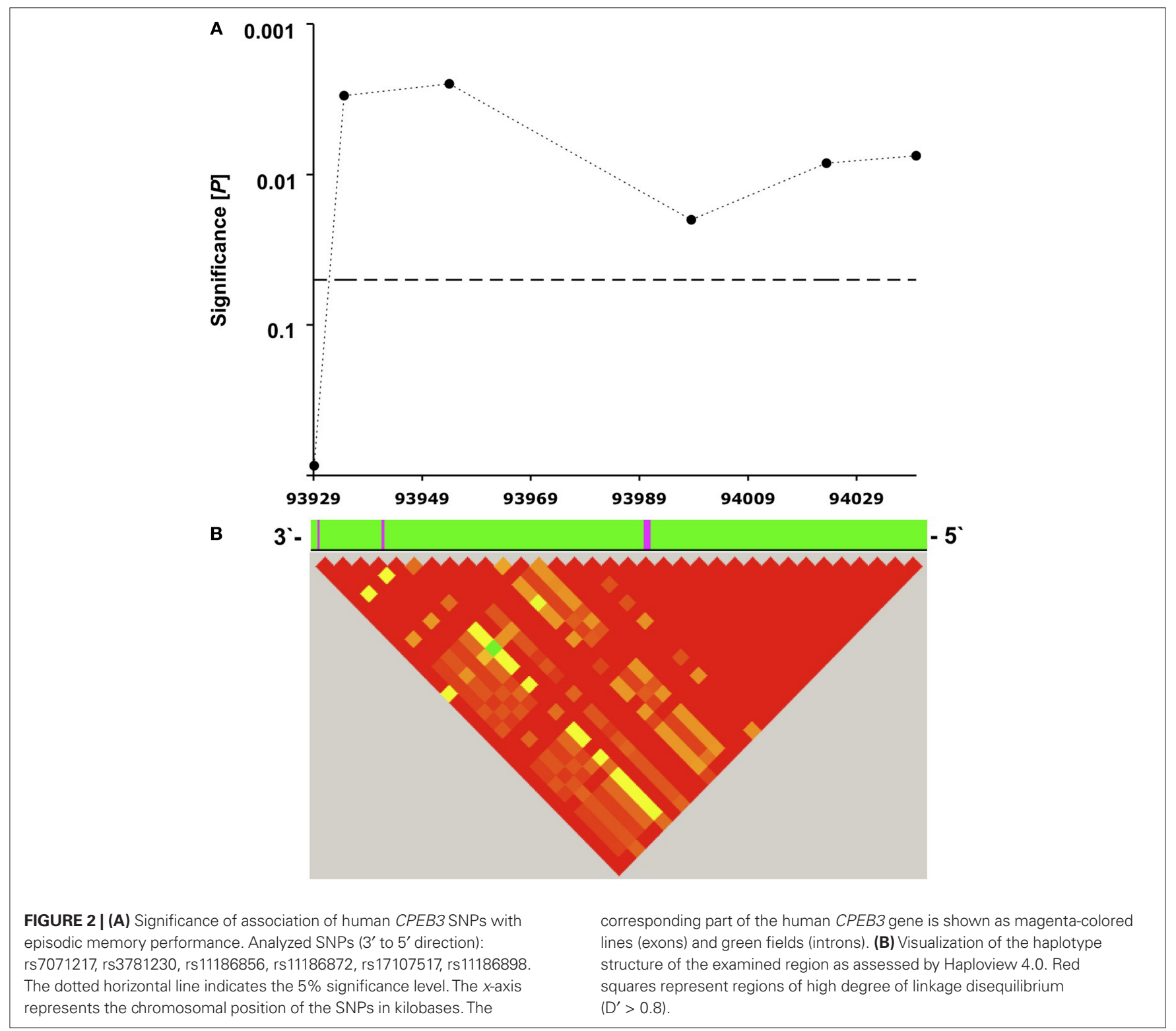


ribozyme sequence had significantly poorer episodic memory performance for words with positive emotional content than $T$ allele carriers of SNP rs11186856 $(2.5 \pm 0.2$ recalled words $5 \mathrm{~min}$ after learning vs. $3.3 \pm 0.1$, respectively; $F=9.3$; $\mathrm{df}=1$; $P=0.002$, Figure $3 \mathrm{~A} ; 2.4 \pm 0.2$ recalled words $24 \mathrm{~h}$ after learning vs. $2.9 \pm 0.1$, respectively; $F=4.7 ; \mathrm{df}=1 ; P=0.03)$. The corresponding significance level for words with negative emotional

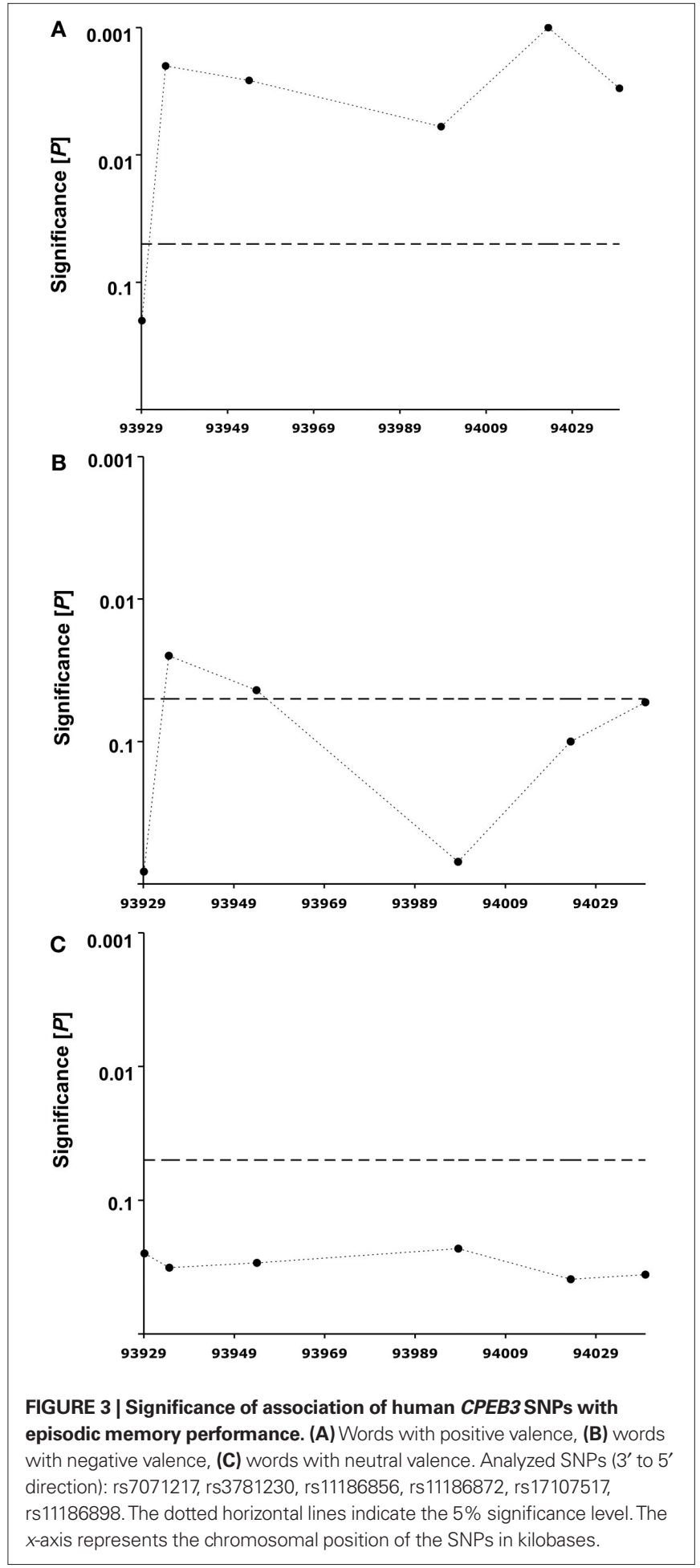

content was weaker (at 5 min: $P=0.025$, Figure 3B; at 24 h: not significant). Interestingly, all of the examined SNPs failed by far to reach significant levels of association for neutral words (Figure 3C).

\section{DISCUSSION}

Behavioral genetic studies have been recently successful in identifying molecules related to human memory functions (de Quervain et al., 2003; Egan et al., 2003; Papassotiropoulos et al., 2006). Significant correlations between genotypic and phenotypic variability point to genes of particular interest and may also identify the precise gene regions related to human memory performance. Here we show that a human-specific polymorphism, a $T$ to $C$ substitution within a highly conserved, small intronic sequence of $C P E B 3$, was associated with decreased episodic memory performance. This intronic sequence has been recently identified as a mammalian conserved, functional CPEB3 ribozyme. Compared to the ancestral sequence, the rare $C$ containing sequence exhibits more than twofold rate of self-cleavage and may therefore result in increased number of truncated CPEB3 pre-mRNAs and ultimately to decreased CPEB3 protein expression (Salehi-Ashtiani et al., 2006). Therefore, the finding of decreased delayed verbal memory performance in homozygous $C$ allele carriers suggests a role for human CPEB3 in this memory task. Furthermore, we found that the genotype effects depend on the emotional valence of the presented material, which suggests that CPEB3 might be involved in neural processes and circuits related to emotional memory, such as the amygdala.

Given the correlative nature of human behavioral genetic studies the question arises as to the specificity of the observed genetic association. Importantly, performance levels in the immediate recall task were identical across $C P E B 3$ genotype groups, which indicates that the genotype-dependent differences in delayed episodic memory were not caused by genotype effects on confounding factors such as motivation, attention or working memory. The fact that $C P E B 3$ was associated with episodic memory performance already 5 min after learning does not contradict the presumed role of $\mathrm{CPEB}$ proteins in long-term memory formation because human shortterm memory performance is affected by preexisting associative networks stored in long-term memory (Stuart and Hulme, 2000). In addition to the ribozyme SNP rs11186856, adjacent SNPs were also significantly associated with memory performance because they are located within one haplotype block. Given the genomic positions of the adjacent SNPs and the absence of any biological data supporting a functional role of these polymorphisms, we assume that the genetic variation causing the observed association is SNP rs11186856.

Taken together, we show that human $C P E B 3$ is associated with delayed verbal memory recall, especially of emotionally arousing information. Further studies are warranted to explore the precise molecular mechanism of this genetic association and its specificity for emotional information.

\section{ACKNOWLEDGMENTS}

This work was supported by grants from the Swiss National Science Foundation to D.Q. and A.P. (PP00B-106708, PP00B-68859, PP00B-114813). 


\section{REFERENCES}

Berger-Sweeney, J., Zearfoss, N. R., and Richter, J. D. (2006). Reduced extinction of hippocampal-dependent memories in CPEB knockout mice. Learn. Mem. 13, 4-7.

de Quervain, D. J., Henke, K., Aerni, A., Coluccia, D., Wollmer, M.A., Hock, C., Nitsch, R. M., and Papassotiropoulos, A. (2003). A functional genetic variation of the 5-HT2a receptor affects human memory. Nat. Neurosci. 6, 1141-1142.

Egan, M. F., Kojima, M., Callicott, J. H., Goldberg, T. E., Kolachana, B. S., Bertolino, A., Zaitsev, E., Gold, B., Goldman, D., Dean, M., Lu, B., and Weinberger, D. R. (2003). The BDNF val66met polymorphism affects activity-dependent secretion of BDNF and human memory and hippocampal function. Cell 112, 257-269.

Hager, W., and Hasselhorn, M. (1994). Handbuch deutschsprachiger Wortnormen. Göttingen, Hogrefe.

Huang, Y. S., Carson, J. H., Barbarese, E., and Richter, J.D. (2003). Facilitation of dendritic mRNA transport by CPEB. Genes. Dev. 17, 638-653.
Huang, Y. S., Kan, M. C., Lin, C. L., and Richter, J. D. (2006). CPEB3 and CPEB4 in neurons: analysis of RNAbinding specificity and translational control of AMPA receptor GluR2 mRNA. EMBO J. 25, 4865-4876.

Keleman, K., Kruttner, S., Alenius, M., and Dickson, B. J. (2007). Function of the Drosophila CPEB protein Orb2 in long-term courtship memory. Nat. Neurosci. 10, 1587-1593.

Klann, E., and Dever, T. E. (2004) Biochemical mechanisms for translational regulation in synaptic plasticity. Nat. Rev. Neurosci. 5, 931-942.

Mendez, R., Hake, L. E., Andresson, T. Littlepage, L. E., Ruderman, J. V., and Richter, J. D. (2000). Phosphorylation of CPE binding factor by Eg2 regulates translation of c-mos mRNA. Nature 404, 302-307.

Papassotiropoulos, A., Stephan, D. A., Huentelman, M. J., Hoerndli, F. J., Craig, D. W., Pearson, J. V., Huynh, K. D., Brunner, F., Corneveaux, J., Osborne, D., Wollmer, M.A.,Aerni,A., Coluccia, D., Hanggi, J., Mondadori, C. R., Buchmann, A., Reiman, E. M.,
Caselli, R. J., Henke, K., and de Quervain,D.J.(2006).Common Kibra alleles are associated with human memory performance. Science 314, 475-478.

Richter, J. D. (2007). CPEB: a life in translation. Trends Biochem. Sci. 32, 279-285.

Salehi-Ashtiani, K., Luptak, A., Litovchick, A., and Szostak, J. W. (2006). A genomewide search for ribozymes reveals an HDV-like sequence in the human CPEB3 gene. Science 313, 1788-1792.

Si, K., Giustetto, M., Etkin, A., Hsu, R. Janisiewicz, A. M., Miniaci, M. C., Kim, J. H., Zhu, H., and Kandel, E. R. (2003a).A neuronal isoform of CPEB regulates local protein synthesis and stabilizes synapse-specific longterm facilitation in aplysia. Cell 115 893-904.

Si, K., Lindquist, S., and Kandel, E. R. (2003b). A neuronal isoform of the aplysia CPEB has prion-like properties. Cell 115, 879-891.

Stuart, G., and Hulme, C. (2000). The effects of word co-occurrence on short-term memory: associative links in long-term memory affect short-term memory performance. J. Exp. Psychol. Learn. Mem. Cogn. 26, 796-802.

Conflict of Interest Statement: The authors declare that the research was conducted in the absence of commercial or financial relationships that could be construed as a potential conflict of interest.

Received: 15 December 2008; paper pending published: 09 January 2009; accepted: 20 April 2009; published online: 04 May 2009

Citation: Vogler C, Spalek K, Aerni A, Demougin P, Müller A, Huynh KD, Papassotiropoulos A and de Quervain DJF (2009) New information about Albert Einstein's brain. Front. Behav. Neurosci. (2009) 3:4. doi: 10.3389/neuro. 08.004.2009

Copyright (c) 2009 Vogler, Spalek, Aerni, Demougin, Müller, Huynh, Papassotiropoulos and de Quervain. This is an open-access article subject to an exclusive license agreement between the authors and the Frontiers Research Foundation, which permits unrestricted use, distribution, and reproduction in any medium, provided the original authors and source are credited. 